\title{
With thanks to our 2011 peer reviewers
}

We are grateful to the following people for their significant contribution to Chronic Diseases in Canada and Chronic Diseases and Injuries in Canada as peer reviewers in 2011. Their expertise ensures the quality of our journal and promotes the sharing of new knowledge among peers in Canada and internationally.

\begin{tabular}{|c|c|c|}
\hline Alexander Allen & Gail Eyssen & John McLaughlin \\
\hline Sten Ardal & Lin Fang & Joel Monárrez-Espino \\
\hline Jon Baio & Stuart Ferguson & Victoria Nadalin \\
\hline Chris Bajdik & Jean-Yves Frappier & Bruce Newbold \\
\hline Christina Bancej & Alain Gauthier & Jillian Oderkirk \\
\hline Cheryl Beach & Patricia Goggin & Louise Parker \\
\hline Philip Bigelow & Carolyn Gotay & Scott Patten \\
\hline Caroline Bois & Gillian Hanley & William Pickett \\
\hline Roxane Borgès Da Silva & Duncan Hunter & Ian Pike \\
\hline Alex Bottle & Jennifer Hutcheon & Ron Plotnikoff \\
\hline Robert Brison & Fiona Johnson & Catherine Rice \\
\hline Brenda Bruner & Ken Johnson & Mylène Riva \\
\hline David Buckeridge & Charlotte Jones & Elizabeth Robinson \\
\hline Ann Burchell & Katherine Kitzmann & Marie-Claude Rousseau \\
\hline Pat Camp & Richelle Koopman & Katreena Scott \\
\hline Yue Chen & Manon Labrecque & Baiju Shah \\
\hline Isabelle Chevalier & Jennifer Landry & Suzanne Smith \\
\hline Mary Chipman & Scott Leatherdale & David Streiner \\
\hline Neil Collishaw & Lynn Lethbridge & Mary Thompson \\
\hline Lori Curtis & Gary Liss & Afshin Vafaei \\
\hline Patrick Daigneault & Julian Little & Carl Van Walraven \\
\hline Carolyn Dayton & Rahul Malhotra & Ron Wall \\
\hline Prithwish De & Heather Manson & Lynne Warda \\
\hline Sarah Dufour & Cora McCloy & Allison Watts \\
\hline Eric Emerson & Kwame McKenzie & Erica Weir \\
\hline Temitope Erinosho & Jennifer McKibbon & Brandy Wicklow \\
\hline
\end{tabular}

